\title{
« La manière de ton parler par personnages »: pratiques et censures de la personnification dans le Débat sur Le Roman de la Rose (1401-1402)
}

\section{Estelle Doudet}

\section{(2) OpenEdition}

Journals

Édition électronique

URL : http://journals.openedition.org/rhetorique/1027

DOI : $10.4000 /$ rhetorique.1027

ISSN : 2270-6909

Éditeur

UGA Éditions/Université Grenoble Alpes

Édition imprimée

ISBN : 978-2-37747-248-2

\section{Référence électronique}

Estelle Doudet, « « La manière de ton parler par personnages »: pratiques et censures de la personnification dans le Débat sur Le Roman de la Rose (1401-1402) », Exercices de rhétorique [En ligne], 15 | 2020, mis en ligne le 11 novembre 2020, consulté le 03 décembre 2020. URL : http:// journals.openedition.org/rhetorique/1027 ; DOI : https://doi.org/10.4000/rhetorique.1027

Ce document a été généré automatiquement le 3 décembre 2020.

\section{(i) (-) (2)}

Les contenus de la revue Exercices de rhétorique sont mis à disposition selon les termes de la Licence Creative Commons Attribution - Pas d'Utilisation Commerciale - Partage dans les Mêmes Conditions 4.0 International. 


\title{
« La manière de ton parler par personnages »: pratiques et censures de la personnification dans le Débat sur Le Roman de la Rose (1401-1402)
}

\author{
Estelle Doudet
}

1 La Briefve Declaration qui explicite les termes rares du Quart Livre s'ouvre par une figure de style: «Prosopopée: desguisement, fiction de persone ${ }^{1}$.» Loin d'éclaircir cette «diction obscure» à l'intention des lecteurs peu versés dans la terminologie rhétorique, l'explication rabelaisienne en accentue le brouillage. On y reconnaît la fictio personarum: commentée depuis les rhétoriciens antiques ${ }^{2}$, la notion englobe au $\mathrm{XVI}^{\mathrm{e}}$ siècle deux tropes aujourd'hui distincts, la prosopopée et la personnification. Mais l'ajout de « déguisement » trouble cette définition déjà complexe. La figure permettrait à ses utilisateurs de mettre en œuvre une stratégie de communication masquée, un jeu de rôles retors exposé dans l'extrait où apparaît le terme commenté :

Semblablement pourroit le medecin ainsi desguisé en face et habitz [...] respondre à ceulx qui trouveroient la prosopopée estrange: «ainsi me suis je accoustré, non pour me guorgiaser et pomper : mais pour le gré du malade, lequel je visite ${ }^{3}$. » Rabelais pointe ici une double difficulté de ce que nous appelons une personnification. Si ce mot n'est devenu usuel qu'en français classique ${ }^{4}$, le fait de "parler en personnages " ou de "faire par personnages ", désignations de la fictio personarum en moyen français, a occupé une place centrale dans les arts de rhétorique dès l'Antiquité. Mais il y a longtemps désigné moins une catégorie précise qu'une constellation de figures interconnectées, l'apostrophe, la prosopopée, la personnification, voire l'allégorie, dont la personnification est l'un des supports fréquents ${ }^{5}$. Outre le caractère peu systématique de sa théorie, la pratique d'une fiction de personne n'est pas sans ambiguïté. La puissance persuasive de la figure tient à la convenance entre les valeurs incarnées par la créature de papier, son comportement et les discours qu'elle adresse à 
un public. Il est donc nécessaire de veiller à ce qu'une parfaite congruence règne entre l'apparence, les mots et le sens des personnifications; sinon, leur fiction pourrait dériver vers la feinte, voire le « desguisement ».

2 Les enjeux et les périls de la personnification ont été beaucoup commentés au tournant du Moyen Âge et de la Renaissance. Entre 1400 et 1460, les publications discutant cette figure se sont multipliées, ce qui ne surprend guère si l'on considère qu'elle était omniprésente dans les œuvres françaises et que l'art rhétorique était d'ores et déjà le plus valorisé des savoirs lettrés. Aux réflexions développées par les théoriciens Jacques Legrand ou l'Infortuné ${ }^{~ s e ~ s o n t ~ a j o u t e ́ s ~ d ' i n t e n s e s ~ d e ́ b a t s ~ m e t t a n t ~ a u x ~ p r i s e s ~ d e ́ f e n s e u r s ~}$ et détracteurs $d u$ "faire par personnage " dans les fictions littéraires. La présente contribution se propose d'éclairer le rôle joué par la critique de la personnification dans le débat sur Le Roman de la rose, la première querelle documentée de la littérature française $e^{7}$. Pourquoi la discussion de cette œuvre romanesque s'est-elle focalisée sur les vertus et les vices attribués à cette figure de rhétorique au début $\mathrm{du} \mathrm{xv}^{\mathrm{e}}$ siècle? Comment les personnifications ont-elles été commentées, fabriquées, censurées par les débatteurs? Quelles nouvelles visions de la relation littéraire, de la responsabilité de l'auteur à l'influence du style sur le lectorat, ont été forgées lors de cette querelle pionnière?

\section{"Le peril du Roumant ${ }^{8}$ ": la Rose mise en débat (1401-1402)}

3 Célèbre pour avoir inauguré la longue histoire des querelles littéraires françaises, le débat sur le Roman de la rose explore une œuvre elle-même fameuse pour sa dynamique contradictoire. L'éducation sentimentale dépeinte par Guillaume de Lorris dans la première partie du roman est en effet déjouée par la continuation satirique offerte dans la seconde partie par Jean de Meun à la fin du XIII ${ }^{e}$ siècle. La polyphonie étourdissante du texte, ainsi que les dissonances obscènes introduites dans la fiction courtoise par les vers ironiques de Jean de Meun, ont d'emblée fait scandale, donnant naissance au fil du $\mathrm{XIV}^{\mathrm{e}}$ siècle à de nombreux commentaires et à des gestes de récriture censurant les épisodes jugés scandaleux ${ }^{9}$.

4 Fallait-il élever la Rose au rang de chef-d'œuvre fondateur et attribuer à Jean de Meun le statut de père d'une littérature française que l'on appellera bientôt moderne ${ }^{10}$ ? C'est autour de cette question que, de l'été 1401 à l'hiver 1402, se sont opposés trois défenseurs du " génie » de Jean de Meun à deux détracteurs du style « vicieux » de son «fol livre ${ }^{11} »$. La querelle épistolaire s'est rapidement cristallisée sur une figure troublante, la personnification.

5 Pendant les premiers échanges, la question du « faire par personnages » est restée un point relativement mineur, le débat ayant porté sur le statut de chef-d'œuvre prêté ou non à la Rose par un lectorat cultivé. À l'été 1401, les administrateurs royaux Jean de Montreuil et Gontier Col ont déclaré inégalables l'inventio ainsi que «la clarté, la propriété et l'élégance» du style de Jean de Meun ${ }^{12}$. Leurs éloges, qui ont d'abord circulé en latin et en français dans le petit cercle des humanistes et hauts fonctionnaires de Charles VI, ont suscité la réponse de Christine de Pizan, elle aussi active à la cour. 
6 S'appuyant sur une lecture minutieuse quoique souvent troublée par les saillies grivoises de Jean de Meun ${ }^{13}$, l'écrivaine a au contraire jugé condamnable cette œuvre vaine et pernicieuse « qui mieulx peut estre appellee droite oysiveté que œuvre utile ». Elle justifie son opinion négative en soulignant l'obscénité de certains passages notamment la défense par Raison d'un langage sans fards, illustré par l'exemple des «couilles»- et en démontrant la misogynie des discours attribués à la plupart des personnages, du Jaloux en délire au prêtre Genius qui prêche l'activité sexuelle à grand renfort de métaphores équivoques ${ }^{14}$. Le commentaire de Christine de Pizan a donc orienté le débat sur certains actants de la polyphonie énonciative créée par Jean de Meun, la Vieille, le Jaloux, Raison, Nature et Genius. Selon elle, la mise en scène de ces fictions de personnes est volontairement viciée, comme le montrent les propos scandaleux prêtés à Raison ${ }^{15}$. En revanche, la constante misogynie exprimée par les personnifications trahirait la voix déguisée de l'auteur, dont l'intention serait de diffamer mensongèrement toutes les femmes ${ }^{16}$.

7 Qualifiées d'« invective » par des adversaires qui n'ont d'abord guère daigné lui répondre ${ }^{17}$, les analyses de Christine ont eu un impact important sur l'évolution de la dispute. Compilé par l'écrivaine et offert à la reine de France Isabeau de Bavière en février 1402, le Livre des epistres du debat sus le Rommant de la Rose a livré au public de cour les premiers échanges épistolaires, poussant deux nouveaux débatteurs à s'engager. En mai-juin 1402, le chancelier de l'Université de Paris Jean Gerson a composé un traité en français sur le roman puis exprimé sa condamnation de l'œuvre dans plusieurs sermons prêchés à Paris à la fin de la même année ${ }^{18}$. Le chanoine Pierre $\mathrm{Col}$, frère de Gontier et collègue de Gerson à Notre-Dame ${ }^{19}$, a réagi à la lecture gersonienne par le biais d'une épître à Christine de Pizan proposant un réexamen minutieux des vices rhétoriques et éthiques repérés chez Jean de Meun. Si Christine a répondu à Pierre $\mathrm{Col}$ en une contre-argumentation circonstanciée, Jean Gerson a mis un point final à la dispute par une lettre envoyée à son collègue pendant l'hiver 1402-1403, lui enjoignant de s'adonner à de plus chastes lectures ${ }^{20}$.

8 L'intervention inattendue de Gerson, à laquelle je vais surtout m'intéresser, a donné au débat deux inflexions majeures. Grâce à lui, la dispute de cour a gagné les milieux urbains et ecclésiastiques; le lexique utilisé pour disqualifier le style de Jean de Meun ainsi que les cadres d'analyse permettant d'évaluer vertus et vices de l'œuvre littéraire en ont été sensiblement modifiés. En outre, Gerson a élargi la critique de Christine contre la misogynie du roman à deux fautes pour lui étroitement liées, le caractère subversif d'une œuvre incitant ses lecteurs à la luxure et la transgression des règles rhétoriques que révèlent les personnifications de Jean de Meun :

Et yci garda mal l'acteur les regles de mon escole, les regles de rethorique, qui sont de regarder cil qui parle, et a qui on parle, et pour quel temps on parle. Et n'est pas le deffault yci seulement, car es autres lieux pluseurs, il attribue a la personne qui parle ce qui ne lui doit appartenir ${ }^{21}$.

\section{"La manière de ton parler par personnages ${ }^{22}$ ": pouvoirs et perversions de la personnification selon Jean Gerson}

9 Seule pièce du débat à nous être parvenue sous la forme d'un traité et non d'une lettre adressée à un contradicteur ${ }^{23}$, le Traictié contre le Roumant de la Rose manifeste 
l'importance donnée par Gerson à la question des figures de style. D'une manière inédite dans la querelle, l'argumentation est confiée à une personnification, Éloquence Théologienne. Incarnation de la rhétorique chrétienne et masque du prédicateur Gerson, elle expose avec précision le fonctionnement de la figure qu'elle-même illustre : la personnification est une «fiction poetique » qui permet de communiquer des idées en "parl[ant] par personnage ». Son omniprésence dans le Roman de la rose autorise à qualifier ce texte de «livre de personnages ", mais pousse à questionner la "propriété » des discours qui sont prêtés par l'auteur à ces innombrables êtres de fiction :

Il a voulu composer un livre de personnages ou quel il fait parler par grant maistrise chascun selon son droit et proprieté.

Cest aucteur ne parle point mais autres qui sont la introduiz ${ }^{24}$.

Le commentaire d'Éloquence atteste d'une solide culture rhétorique, mise en valeur par des références classiques comme l'Épître aux Pisons d'Horace ${ }^{25}$. Elle permet d'étayer la critique du vicieux « parler par personnages » mis en œuvre dans la Rose et de montrer l'impact potentiellement désastreux que pourrait avoir ce texte sur un public non averti.

10 L'un des principaux « deffault[s] » du roman, déjà pointé par Christine de Pizan, est le choquant décalage entre l'identité des créatures personnifiées et les discours qu'elles énoncent. L'Institution oratoire de Quintilien comme la Rhétorique à Herennius - deux sources bien connues au début $d u \mathrm{xv}^{\mathrm{e}}$ siècle ${ }^{26}-$, ont insisté sur l'importance de la conformatio, ou fabrication d'une forma, pour reconfigurer un élément inanimé ou virtuel sous la forme d'un personnage aux traits et aux comportements humains. Ils l'ont articulée à la sermocinatio, le don de la parole à des êtres absents ou muets. La convenance entre le caractère du personnage créé et ses paroles est essentielle d'un point de vue éthique comme stylistique. Les arts de rhétorique contemporains du débat soulignent avec force cette nécessité. L'Instructif de seconde rhétorique y insiste dès le début du chapitre consacré aux techniques du «faire en personnages » :

S'en personnaiges l'on veult faire [...]

Puis considerer quelle forme

À chascun convient assorter

Selon qu'el' peut estre conforme ${ }^{27}$.

Mais la Rose a introduit de dangereuses turbulences dans cette règle fondamentale. Les défenseurs du roman, comme plus tard les critiques modernes ${ }^{28}$, ont vu une innovation géniale dans l'autonomie discursive maximale que Jean de Meun a offerte à ses créatures de papier. Chaque entité mise en scène assume en effet son propre discours. Il n'y a donc a priori rien d'inconvenant à ce que le Jaloux éructe un discours soupçonneux ou que la Vieille insinue mielleusement ses conseils d'entremetteuse, rôle qu'elle assume depuis Ovide ${ }^{29}$. Mais selon Christine et Gerson, l'argument est faux car ce supposé respect de la convenance ne s'étend pas à l'ensemble des personnages du roman. Un principe majeur de la rhétorique, la virtus dicendi (la force de l'expression, ici assurée par sa cohérence ${ }^{30}$ ), est même systématiquement bafoué dans le cas des personnifications. Nature, figuration traditionnelle de la perpétuation du genre humain, disserte de prescience divine, tandis que son chapelain Genius argumente en faveur d'une sexualité sans frein ${ }^{31}$. Une éventuelle méconnaissance du sens originel de ces figures ne peut nullement être plaidée puisque Jean de Meun les emprunte à des sources célèbres. Nature tire ainsi ses traits de la protagoniste du De Planctu Naturae d'Alain de Lille. Toutefois le philosophe du XII ${ }^{e}$ siècle s'était gardé de faire énoncer à son 
personnage un discours moralement et stylistiquement inadéquat. La corruption est donc le fait du romancier :

Ceste fiction poetique fut corrumpuement estraite du grant Alain [...]. Par personnage quelconque il ne parla onques en telle maniere ${ }^{32}$.

Les inconvenances irrévérencieuses de la Rose atteignent leur point de rupture avec la mise en scène de Raison. "Raison la sage » est transformée en "Raison la rage ", militante d'une parole sexuelle libérée. Éloquence s'en indigne :

Comment donques se peut soustenir bailler a dame Raison un tel personnage, ainsi comme ceulx qui ainsi ne le font feussent hors du sens et de raison, comme parlast Raison, non mie la sage, mais l'assotee et la souillarde? En nom Dieu, ce personnage eust mieulx appartenu a pourceaux ou a chiens que a Raison ${ }^{33}$.

Conséquence de la rupture entre leur forma et leur sermo, les personnifications du roman reviennent, par un vicieux détour, à l'un des sens étymologiques de prosopopeia : elles se font masques de théâtre, feintes, mensonges. Nature, Raison et d'autres figures familières au lectorat médiéval semblent gagnées par l'influence de Faux Semblant, cette créature dérangeante à laquelle Jean de Meun a donné la capacité de tenir n'importe quel discours en assumant n'importe quel statut ${ }^{34}$.

Une autre perversion tient au brouillage des modes d'énonciation. Jacques Legrand, qui théorise l'œuvre de fiction (poetrie) au moment où commence le débat sur le Roman de la rose, a rappelé les possibilités traditionnelles à la disposition des écrivains :

En poetrie le poete aucunefoiz parle tout seul, et ainsi fait Virgille en ses Georgiques, autrement dictes caracteres; et aucunefois le poete ne parle point ne mais en personne d'autrui, et lors celles poetries sont nommees dragmatiques, et de ceste façon communement sont comedies et tragedies; et aucunefois le poete parle en une partie et non mie en l'autre, et ainsi fait Virgille en son livre nomme Eneydos ${ }^{35}$.

Le Roman de la rose oscille entre le mode narratif - dans la partie attribuée à Guillaume de Lorris, le récit est confié à un unique protagoniste, l'Amant, dont le point de vue oriente l'ensemble de l'interprétation - et le mode « dragmatique » que favorise Jean de Meun, diffractant points de vue et paroles entre de nombreuses instances aux opinions divergentes. Pour Legrand, l'énonciation dramatique est le terrain le plus favorable à la pratique des personnifications, mais il faut «faire fictions fondees en raison et en la semblance des choses dont on veut parler ${ }^{36} »$. Or Jean de Meun a multiplié dans un apparent désordre les personnifications travaillées de contradictions, de sorte que l'elocutio de ces personnages et la dispositio générale du texte en sont brouillées. Les lecteurs se trouvent confrontés, juge Gerson, à « un chaos informe, une vraie tour de Babel $^{37}$ ».

Un autre vice tient au caractère insaisissable de l'intention d'auteur dans ce livre protéiforme. La voix auctoriale n'est pas absente du Roman de la rose, mais il est très difficile de déceler les moments où les personnifications parlent en leur nom (in propria persona) et ceux où elles relaient le point de vue du romancier, qui s'exprime alors in persona aliorum :

Je vouldroie bien que ce fol amoureux ne eust usé de ces personnages fors ainsi que

la saincte escripture en use [...], tellement que chascun eust apperceu le reproche $\mathrm{du}$ mal et l'approbacion du bien [...]. Mais nennil voir. Tout semble estre dit en sa personne, tout semble estre vray comme euvangille ${ }^{38}$.

Les récepteurs sont donc confrontés à un tourbillon de discours, présentés comme vrais mais potentiellement faux, derrière lesquels se cache une intentio auctoris impossible à cerner. 
Pour les débatteurs qui défendent la virtuosité de Jean de Meun, cette instabilité ne doit pas forcément être analysée comme un défaut. Jean de Montreuil considère qu'un tel jeu sur les limites de la personnification est une licence permise à l'auteur satirique, dont le but est d'inciter au redressement en montrant les dysfonctionnements du discours ${ }^{39}$. Pierre Col ajoute que les alternances d'apparition et de disparition de la voix auctoriale sont à mettre en rapport avec le savant tissage des opinions contraires que Jean de Meun dit pratiquer ${ }^{40}$; mais si Jean n'a été qu'un compilateur, comment le considérer comme un grand auteur?

Pour les détracteurs, tout cloche dans l'œuvre. L'image de la boiterie parcourt les échanges de 1402. Contrairement à Montreuil et Col, qui interprètent les vices d'écriture comme une leçon adressée aux récepteurs - « on doit ramentevoir le pié de quoy on cloche pour plus droit aller ${ }^{41} »-$, Christine de Pizan et Gerson voient dans la claudication rhétorique une incitation à la faute morale. Dans ses sermons, Gerson rapproche les personnages instables du roman de Luxure, elle aussi dépeinte en boiteuse « qui fait clochier et boiter sur le chemin des vertus ${ }^{42}$ ». Pour le prédicateur, le responsable de cet appel général à la boiterie ne saurait être que Jean de Meun, qui a signé son texte du nom de Clopinel (le boiteux ${ }^{43}$ ).

\section{"Mieulx ensevelissement de feu que couronne de laurier $^{44} »$ : censurer une œuvre, repenser la littérature}

Malgré la proximité de leurs critiques, Christine et Gerson se sont positionnés différemment sur l'issue du débat. Interpellée par le chanoine Col après la publication du Traité de Gerson en mai 1402, l'écrivaine a accepté de reprendre la dispute épistolaire entamée l'année précédente. Elle souligne toutefois la difficulté de conclure une querelle d'interprétation littéraire, par nature sans fin :

Scez tu comme il va de celle lecture? Ainsi comme des livres des archemistes : les uns les lisent et entendent d'une maniere, les autres qui les lisent, les entendent tout au rebours, et chacun cuide trop bien entendre; et sur ce ilz oeuvrent et apprestent fourniaulx a lembis et croisiaulx, et entre meslent divers metaulx et matieres et soufflent fort, et, pour un petit de sublimacion ou congiel qui leur appert merveillable, ils cuident ataindre a merveilles. [...] Ainsi est il de toy et de moy et de plusieurs : tu l'entens et le prens d'une maniere, et moy tout au rebours ; tu recites, je replique; et quant nous avons fait et fait, tout ne vault riens, car la matiere est tres deshonneste, ainsi comme aucuns arquemistes qui cuident faire de fiens or ${ }^{45}$.

À travers la malicieuse métaphore des alchimistes se dessine en creux un ultime portrait de Jean de Meun, magicien qui a déployé dans la Rose un style virtuose transgressant les normes du langage socialement autorisé. Ses commentateurs sont campés en apprentis sorciers, affairés à concocter de savants systèmes de lecture à partir d'une œuvre de fiction contestable. Christine laisse ouverte la discussion. Son ironie souriante s'explique sans doute par les gains manifestes qu'elle a tirés de la querelle. Outre le prestige donné par sa participation à une dispute publique entre lettrés, elle a reçu à cette occasion la réputation inédite d'une autrice défendant la vertu des femmes et les pouvoirs éthiques de la littérature ${ }^{46}$.

19 Les réactions de Gerson reflètent une autre position. Théologien de profession, il s'est sans doute agacé de voir qualifier Jean de Meun de «tres devolt catholique et eslevé theologien", de "tres excellant et inreprehensible docteur en sainte divine 
Escripture $^{47} »$. Ses choix d'écrivain ont été bafoués par le dévoiement de la personnification de Raison, celle-ci étant une figure centrale de l'œuvre gersonienne où elle incarne la bonne éducation chrétienne ${ }^{48}$. Enfin, la personne de Gerson n'a guère été épargnée. Des plaisanteries égratignant en lui l'ecclésiastique et le styliste ont circulé de la chancellerie royale à Notre-Dame de Paris. Jean de Montreuil a raillé la compréhension réduite qu'un «homme impropre à la perpétuation de l'espèce humaine " pouvait avoir d'une fiction faisant l'apologie de la sexualité; Pierre Col a moqué les sermons sur la Rose prêchés par un prédicateur bien innocent en ces matières : «j'oseroie dire qu'il contoit mieulx la Trinité qu'il ne fait Fol Amoureux ${ }^{49}$ ». Il a également été insinué que les personnifications du Traité ne respectaient pas les règles $\mathrm{du}$ « faire par personnage $\mathrm{e}^{50}$ ». Ces petites perfidies, qui relèvent de la libertas disputandi chère aux humanistes ${ }^{51}$, éclairent la posture particulière choisie par Gerson évaluateur de la Rose: celle d'un orateur chrétien qui soupèse les figures à l'aune de leurs vertus morales; d'un homme de cour et d'église qui juge de la communication littéraire dans une perspective civique et spirituelle; d'un censeur dont le métier est de traquer les déviances et d'extirper les faux discours de l'espace public ${ }^{52}$.

Pareilles attitudes transparaissent dans la manière dont le Traité a instrumentalisé la personnification. Plutôt que d'attaquer ad hominem le défunt Jean de Meun ${ }^{53}$, Gerson a donné à sa cible les traits d'un être de fiction. Le Fol Amoureux incarne l'auteur incriminé mais aussi l'Amant, principal personnage du roman, et plus généralement les manipulateurs qui se cachent derrière des fictions de discours pour faire scandale sans assumer leurs actes :

Se aucun se nommoit adversaire du roy de France et sus ce nom et comme tel lui faisoit guerre, ce nom le garderoit il d'estre traitre et de la mort? [...] Se en la personne d'un herite ou d'un Sarrazin, voire du deable, aucun escript et seme erreurs contre la crestienté, en sera il excusé ${ }^{54}$ ?

La pluri-référentialité du Fol Amoureux permet à Gerson de déplacer le débat de l'écriture romanesque vers une réflexion sur la place de la fiction dans la communication politique et religieuse, domaines où les figures de rhétorique démontrent leurs plus fortes capacités d'action et leurs plus grands dangers de subversion.

21 Cette approche, qui fait de l'œuvre une action publique et donne à l'auteur la responsabilité de l'ordre ou du désordre social, juge la valeur d'un style à son impact sur les récepteurs. Dans la Rose, les discours obscènes de Raison sont adressés à un Amant qui ne les comprend pas, à l'inverse de ce qu'auraient pu faire « un saige clerc [...] ou un grant theologien ", moins prompts à avoir l'imagination enflammée par ces évocations tendancieuses et plus aptes à les décrypter ${ }^{55}$. Il en est de même des publics réels. La capacité des lecteurs à saisir les dissonances des personnifications est âprement disputée par les débatteurs. À Pierre Col qui cite un ami guéri de sa lubricité par la lecture de la $\operatorname{Ros}^{56}$, Christine de Pizan réplique par le cas inverse d'un mari dont la jalousie fut attisée par le roman au point d'user de violence contre son épouse :

Un homme marié, lequel ajouste foy au Rommant de la rose comme a l'Euvangile. [...]

Et a chacun mot qu'il treuve a son propos, il fiert un coup ou deux du pié ou de la paume : si m'est avis que, quiconque s'en loue, celle povre femme le compare cher ${ }^{57}$. réactions tout en s'adressant à des instances capables de le contrôler : «il publia son livre a jeunes gens qui en abusoient. [...] Ostez, bonnes gens, ces livres d'entre vos filles 
et enfans ${ }^{58}$." Jeunes gens et jeunes filles, lecteurs habituels des romans, seraient les plus vulnérables au style trompeur de Jean de Meun :

$\mathrm{Au}$ feu! Bonnes gens, au feu! [...] Qui ne fuit le peril il y trebuchera et y sera pris, comme rat au lardon et le loup en la louviere, ou le papillon au feu de la chadeille pour sa clarté, ou les fols ou les enfans aux espees nues ou aux charbons vifs pour leur beautés.

Or parmi les figures privilégiées de la Rose, la personnification, par nature "véhémente " selon Cicéron ${ }^{60}$, est porteuse d'une puissance d'émotion périlleuse pour ces lecteurs peu avertis. On comprend dès lors pourquoi, sous l'influence de Gerson alias Éloquence Théologienne, le lexique disqualifiant mobilisé contre le roman s'est fait plus agressif.

Dès 1401, Christine de Pizan a condamné dans le texte une "exortacion de vice confortant vie dissolue [...], honte de plusieurs personnes et peut estre d'erreur ${ }^{61} »$. Mais l'hérésie (« erreur ») est restée conditionnelle sous la plume de la poétesse, qui ne prétendait pas en juger. Dans les prêches et le Traité de 1402, ses «deffaults » font du roman "venimeuse doctrine, destruction et desolacion de povres ames crestiennes, illicite perdicion de temps precieux ${ }^{62} »$. Trompeur comme l'est la lame cachée sous le miel ou l'hameçon masqué sous le brillant du leurre, le livre est «blaspheme et heresie »; il doit être "expulsé de notre république de religion chrétienne ${ }^{63}$ ». À ses ouailles, l'orateur Gerson déclare envisager l'autodafé des exemplaires en sa possession et la censure des autres :

Se j'avoie ung Roumant de la Rose qui feust seul et vaulsist mil livres, je l'ardroie plus tost que je ne le vendisse [...]. Se je savoie que il [Jean de Meun] ne s'en fust repenti, je ne prieroie pour lui nez que pour Judas [...]. Se je confessoie personne qui en abusast, je lui commanderoie effacer plusieurs choses, ou du tout le gecter hors ${ }^{64}$.

Aucun Roman de la Rose n'a été brûlé en 1401-1402. Cette surenchère verbale témoigne de la dynamique inquisitoriale des échanges, chaque commentateur cherchant à convaincre les autres de leurs erreurs ${ }^{65}$. Elle démontre surtout l'importance publiquement donnée à un débat qui, de discussion d'un roman à succès, s'est élargi jusqu'à devenir une relecture globale de la fiction littéraire, de ses auteurs et de ses récepteurs.

La première querelle littéraire française a été un débat sur la rhétorique, ses outils, ses normes, ses enjeux. S'il a été essentiellement étudié jusqu'ici par des médiévistes et dans la perspective du genre puisque la question de l'expérience féminine de la littérature y est centrale, le Débat sur le Roman de la rose mérite de retrouver toute sa place dans l'histoire de la rhétorique et de ses renaissances. Il illustre en effet le rhetorical turn du tournant des $\mathrm{XIV}^{\mathrm{e}}$ et $\mathrm{XV}^{\mathrm{e}}$ siècles, changement de paradigme majeur qui, au-delà du cas particulier de l'Italie, a reconfiguré les champs littéraires de la plupart des pays d'Europe. Avant " l'âge de l'éloquence » des XVI ${ }^{\mathrm{e}}-\mathrm{XVII}{ }^{\mathrm{e}}$ siècles s'est développé en France un «âge des orateurs » qui a repensé les statuts d'auteurs, les choix de style et les rôles des publics sous l'angle de la communication oratoire ${ }^{66}$. Dans ce contexte, les figures ont été analysées moins comme des ornements du discours que comme des pratiques performatives capables d'assurer ou de déstabiliser l'espace public. Les personnifications créées par Jean de Meun ont donc été au cœur du débat. Dotées d'une autonomie qui masque l'intention de leur auteur, travaillées de décalages entre ce qu'elles sont et ce qu'elles disent, porteuses de discours inconvenants, ces entités ont 
radicalement remis en cause les principes du "faire par personnages ». Coup de génie ou scandaleuse transgression? Si Jean de Montreuil, Gontier et Pierre Col, Christine de Pizan ont pris position sur cette question, c'est toutefois Jean Gerson qui en a proposé l'approche la plus originale, en articulant usage personnel de la figure de rhétorique et censure publique de son potentiel scandaleux. Il a fabriqué Éloquence Théologienne pour contrer les jeux de masque de Faux Semblant, dressé sa sage Raison contre la folle Raison du roman, promis la Rose au feu et finalement usé de son autorité pour couper court à la querelle des interprétations ${ }^{67}$.

En proposant d'évaluer la fiction romanesque à l'aune de la rhétorique chrétienne, Gerson a indéniablement opéré un coup de force. Certains critiques n'ont pas hésité à comparer son interprétation de la Rose aux lois bannissant le hate speech aux États-Unis, le débat $\mathrm{du} \mathrm{xv}^{\mathrm{e}}$ siècle trouvant une nouvelle actualité dans l'affrontement des détracteurs et des défenseurs du politiquement correct à la fin $\mathrm{du} \mathrm{xx}^{\mathrm{e}}$ siècle $^{68}$. Mais on peut aussi être frappé par la manière dont Gerson a lui aussi joué des défauts de ses personnifications. S'il argumente qu'Éloquence, "avocat de la court chretienne », est un personnage pertinent pour lire un roman d'amour et que son discours, "a voix resonnant doulce et moienne ", n'a pas la véhémence d'une censure ${ }^{69}$, Gerson ne dit rien d'une flagrante faute de genre : la personnification, déguisement du prédicateur, est constamment désignée au masculin. L'impropriété n'a pas échappé à Christine de Pizan, qui s'est emparée de la féminité d'Éloquence pour faire corps avec elle ${ }^{70}$.

$\mathrm{Au}$ début $\mathrm{du} \mathrm{xv}^{\mathrm{e}}$ siècle, alors que le triomphe de la rhétorique redéfinit les normes et les usages des figures de style, la littérature commence insensiblement à changer de genre : orateur chrétien pour Gerson, Éloquence est déjà, pour Christine, femme de lettres ${ }^{71}$.

\section{NOTES}

1. Fr. Rabelais, Le Quart Livre, CEuvres complètes, éd. M. Huchon, Paris, Gallimard, «La Pléiade », 1994, p. 703.

2. Entre autres, Quintilien, Institution oratoire, IX, II, 29, éd. et trad. J. Cousin, Paris, Les Belles Lettres, 1978, t. V ; Rhétorique à Herennius, IV, 66, éd. et trad. G. Achard, Paris, Les Belles Lettres, 1997.

3. Dédicace à Odet de Chastillon, Le Quart Livre, op. cit., p. 518.

4. «Personnifier » devient usuel à partir des Réflexions critiques sur quelques passages du rhéteur Longin de Boileau en 1674; le substantif "personnification» est employé au XVIII siècle (M. Demaules, « Avant-propos », La Personnification du Moyen Âge au XVIII siècle, Paris, Classiques Garnier, 2014, p. 16-18).

5. Si la prosopopée désigne usuellement aujourd'hui l'attribution d'un discours à des êtres absents ou non humains, la personnification donne corps et vie à des éléments abstraits ou inanimés placés dans une relation humaine d'interlocution (G. Molinié, Dictionnaire de rhétorique, Paris, Librairie générale française, 1992, p. 280, 269). 
6. J. Legrand, L'Archiloge Sophie et Livre des bonnes mœurs (vers 1399), éd. E. Beltran, Paris, Honoré Champion, 1986 ; L'Instructif de la seconde rhétorique (vers 1460), dans La Muse et le compas, poétiques à l'aube de l'âge moderne, dir. J.-Ch. Monferran, Paris, Classiques Garnier, 2015, p. 13-194.

7. Existent actuellement deux éditions de référence du débat, chacune avec un titre spécifique : Chr. de Pisan, J. Gerson, J. de Montreuil, Gontier et P. Col, Le Débat sur le Roman de la Rose, éd.

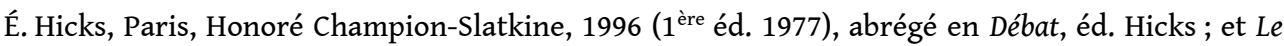
Livre des epistres du debat sus le Rommant de la rose, éd. A. Valentini, Paris, Classiques Garnier, 2016, abrégé en Livre des epistres, éd. Valentini. Les textes français sont cités dans l'édition Valentini, les textes latins dans l'édition Hicks. Les traductions sont miennes. Voir aussi Le Débat sur le Roman de la rose, trad. V. Greene, Paris, Honoré Champion, 2006.

8. J. Gerson, sermon contre la luxure (décembre 1402), Euvres complètes, éd. P. Glorieux, TournaiRome- New York, Desclée \& C $C^{\text {ie }}, 1966$, t. VII*, nº 370, p. 831.

9. P.-Y. Badel, Le Roman de la rose au XIVe siècle, étude de la réception de l'œuvre, Genève, Droz, 1980.

10. En 1511, Jean Lemaire de Belges déclare Jean de Meun premier des " poetes, orateurs et historiiens de la langue françoise, tant antiques que modernes " (La Concorde des deux langages, éd. J. Frappier, Paris-Genève, Droz, 1947, p. 4).

11. «Ingenium actoris » : J. de Montreuil, épître Cum ut dant, dans Débat, éd. Hicks, p. 28 ; J. Gerson, Traité, dans Livre des epistres, éd. Valentini, p. 306, 324.

12. «In inventione nichilominus atque claritate proprietate et elegantia [...] longius anteponens » [«bien supérieur [...] dans le domaine de l'invention comme pour la clarté, la propriété et l'élégance du style »], J. de Montreuil, épître Quo magis, dans Débat, éd. Hicks, p. 29-30.

13. Elle indique avoir lu le roman "au lonc et au lé », en long et en large, mais ajoute avoir dû «passer oultre comme coq sur brese» sur certains vers choquants (Livre des epistres, éd. Valentini, p. 157). Pour défendre sa légitimité, la commentatrice allègue sa maîtrise de la lecture savante pratiquée par les intellectuels auxquels elle s'adresse, tout en se défendant, en veuve vertueuse, d'avoir lu les extraits obscènes qu'elle discute.

14. «Coilles est biaus nons et si l'ains » [ Couilles est un beau mot et je l'aime»] dit Raison (v. 7112) ; «Arez, pour Dieu, baron, arez/ et vos lignages reparez » [ Labourez, par Dieu, labourez, barons, et restaurez vos lignages » »] dit Genius v. 19705-6 : dans Guillaume de Lorris et Jean de Meun, Le Roman de la rose, éd. et trad. A. Strubel, Paris, Le Livre de poche, "Lettres gothiques ", 1992, p. 394, 1022.

15. «Je ose dire que la Raison maistre Jehan de Meun renya son pere a cellui mot » [ " la Raison de maître Jean de Meun a renié son père en prononçant ces mots »] : la personnification de la sagesse se renie en multipliant les énoncés immoraux (Livre des epistres, éd. Valentini, p. 158).

16. S. Huot, "Confronting misogyny: Christine de Pizan and the Roman de la Rose", Translatio Studii. Essays by His Students to Karl D. Uitti, éd. R. Blumenfeld-Kosinski, K. Brownlee, M. Speer et L. Walters, Amsterdam, Rodopi, 2000, p. 169-187.

17. L'humaniste Jean de Montreuil, grand amateur de disputes latines à la virilité surjouée, s'est montré peu à l'aise face au débat en français proposé par la poétesse. Il a laissé Gontier Col lui répondre (Livre des epistres, éd. Valentini, p. 152) : "Tu as nouvellement escript par maniere de invective contre ce que mon maistre Jehan de Meun [...] fist et compila ou livre de la Rose». Col, collègue du défunt mari de Christine, a ensuite prié celle-ci (ibid., p. 169-170) «d'amender de l'erreur et magnifeste folie et demence trop grant a toy venue par presompcion ou outrecuidance et comme femme passionnee en ceste matiere » [« de t'amender de l'erreur, de la folie évidente, de la démence qu'ont suscitées en toi la présomption ou l'arrogance d'une femme passionnément engagée dans cette affaire »].

18. J. Gerson, sermons Poenitemini contre la luxure et sur la chasteté (décembre 1402), CEuvres..., t. VII*, op. cit., n ${ }^{\circ} 370,371,372$, p. 822-851. Pour l'influence du débat sur l'œuvre de Gerson, voir R. Blumenfeld-Kosinski, "Jean Gerson and the Debate on the Romance of the Rose ", A Companion to Jean Gerson, éd. B. P. McGuire, Leyde, Brill, 2006, p. 317-356. 
19. J. L. Baird, «Pierre Col and the Querelle de la Rose», Philological Quarterly $\mathrm{n}^{\circ} 60,1981$, p. 273-286.

20. Gerson, épître Talia de me à Pierre Col, dans Débat, éd. Hicks, p. 175.

21. Gerson, Traité, dans Livre des epistres, éd. Valentini, p. 322 [« Ici l'auteur a mal observé les règles de mon école, les règles de la rhétorique, qui consistent à prendre en compte qui parle, à qui l'on parle et à quel moment. Et ce défaut n'apparaît pas seulement ici ; dans plusieurs autres passages, il attribue au personnage qui parle un discours qui ne lui convient pas. »].

22. Ibid., p. 306.

23. Le traité français rédigé par Jean de Montreuil à la gloire de Jean de Meun, texte déclencheur de la querelle, n'a pas été conservé.

24. Gerson, Traité, dans Livre des epistres, éd. Valentini, p. 303, 311 [« Il a voulu composer une œuvre peuplée de personnifications, où il fait parler chacune, avec une grande virtuosité, de la manière qui lui convient »; « l'auteur ne parle pas mais donne la parole à d'autres »].

25. Éloquence compare la fin obscène du Roman de la rose, qui montre la pénétration du bâton de l'Amant-pèlerin jusqu'au cœur du rosier, à la chimère horacienne « qui fait une tres belle femme ou chief et fenist en poisson » (ibid., p. 316). L'Art poétique d'Horace, qui s'ouvre sur une liste des vices de style, est également cité dans les lettres de Jean de Montreuil et de Pierre Col, qui partagent la culture rhétorique de Gerson. Dans sa dernière lettre à Pierre Col, Gerson ajoutera avoir lu attentivement Boèce, Martianus Capella et Alain de Lille, auteurs qui ont fait de la personnification une figure centrale de l'écriture médiévale.

26. Ils avaient peut-être également connaissance des arts poétiques médio-latins, comme celui de Geoffroi de Vinsauf, qui ont valorisé la personnification au moment où celle-ci prenait son essor dans les productions littéraires en français. Voir Les Arts poétiques du XII et XIII siècles. Recherches et documents sur la technique littéraire au Moyen Âge, éd. E. Faral, Genève-Paris, Champion-Slatkine, 1982 [ 1 ère éd. 1924] ; D. James-Raoul, «La personnification dans les arts poétiques médio-latins des XII ${ }^{\mathrm{e}}$ et XIII ${ }^{\mathrm{e}}$ siècles ", La Personnification, op. cit., p. 35-51.

27. L'Instructif de la seconde rhétorique, op. cit., p. 134, v. 1941, 1946-8 [" Si l'on souhaite faire une œuvre en personnages, il faut considérer quelle forme de discours il convient d'assortir à chacun afin qu'elle puisse être en conformité avec eux »]; voir E. Buron, «Faire en personnages. De la théorie de l'Instructif à la pratique du Jardin de Plaisance ", Cahiers de Recherches Médiévales et Humanistes (CRMH) n ${ }^{\circ} 21,2011$, p. 205-223.

28. Entre autres M.-R. Jung, Étude sur le poème allégorique en France au Moyen Âge, Berne, Francke, 1971 ; A. Strubel, La Rose, Renart et le Graal. La littérature allégorique en France au XIII siècle, Paris, Honoré Champion, 1989; A.J. Minnis, Magister Amoris. The Roman de la Rose and Vernacular Hermeneutics, Oxford, OUP, 2001.

29. Le Traité de Gerson dépeint les défenseurs de la Rose sous les traits d'une foule anonyme ( « une grant tourbe et une flotte, gens sans nombre, jeunes et vieulx, de tous sexes et de tous aages ", dans Livre des epistres, éd. Valentini, p. 302). Toutefois est distingué un adversaire « des plus avisié » qui souligne que Jean de Meun a respecté l'accord entre « la condicion » de certains personnages de fiction (le Jaloux, Vénus) et le caractère "legier» de leurs discours. Ce fin contradicteur est Jean de Montreuil, qui développe ces arguments dans son épître Ut sunt mores (dans Débat, éd. Hicks, p. 42-43) : «qui de personatuum varietate non discernunt, seu notant quibus passionibus moveantur aut induantur affectibus» [«ils ne regardent pas à la diversité des personnages, ils ne remarquent ni de quelles passions ceux-ci sont agités, ni les sentiments qu'il convient de leur prêter »].

30. Les vices décrits par les traités de rhétorique relèvent en général deux types de faute : contre la qualité de l'expression (virtus dicendi) et contre la logique des genres discursifs (genera dicendi); C. Barbafieri et J.-Y. Vialleton, «Introduction », Vices de style et défauts esthétiques, XVI ${ }^{e}-\mathrm{XVI}{ }^{e}$ siècles, Paris, Classiques Garnier, 2017, p. 7-20. 
31. Christine de Pizan commente ironiquement ce dysfonctionnement dans sa réponse à Pierre Col (dans Livre des epistres, éd. Valentini, p. 191) : « il failli a bien introduire ses personnages, de commettre a aucun autre chose que leur office, comme a son prestre qu'il appelle Genius qui tant commande de coucher avec les femmes [...]; si ne sçay a entendre qu'il appartiengne a son office." [ «Il a échoué à présenter correctement ses personnages, dans la mesure où il leur impose de faire autre chose que leur rôle, par exemple son prêtre, nommé Genius, qui ordonne de coucher avec les femmes ; [...] je n'arrive pas à saisir en quoi ce discours correspond à son rôle. »] 32. Gerson, Traité, dans ibid., p. 318 ["Cette fiction poétique s'inspire de manière corrompue d'Alain de Lille. [Mais celui-ci] ne tint pas ces discours par le truchement de n'importe quelle personnification. »].

33. Ibid., p. 320 ["Comment donc peut-on justifier de faire jouer à dame Raison un tel personnage, et soutenir que ceux qui ne l'imitent pas sont des fous et des insensés, quand on fait parler Raison non comme une sage, mais comme une folle et une souillon? Au nom de Dieu, un tel personnage convient mieux à des pourceaux ou à des chiens qu'à Raison. »].

34. Le faux moine Faux Semblant change constamment d'identité sociale et «sai $[t]$ par cuer trestouz langages" (Le Roman de la rose, op. cit., p. 596, v. 11200) ; voir A. Strubel, «De FauxSemblant à Fauvel : la limite de la personnification ", La Personnification, op. cit., p. 109-128.

35. J. Legrand, Archiloge Sophie, op. cit., p. 151-152 [«Dans une œuvre de fiction, le poète parfois parle en son nom, ainsi que le fait Virgile dans ses Géorgiques, aussi dits caractères. Parfois, ce n'est pas lui qui assume la parole, mais un autre personnage, et ces œuvres sont alors nommées dramatiques - c'est le cas des comédies et tragédies. Et parfois le poète assume la parole dans une partie de l'œuvre et non dans l'autre, comme le fait Virgile dans l'Énéide »].

36. Ibid., p. 149.

37. Gerson, épître Talia de me à Pierre Col, dans Débat, éd. Hicks, p. 168.

38. Gerson, Traité, dans Livre des epistres, éd. Valentini, p. 312 [ «Je voudrais bien que ce fol amoureux eût utilisé ses personnages comme le fait la Sainte Écriture [...], afin de permettre à chacun de distinguer la critique du mal et l'approbation du bien. [...] Mais il n'en est rien. Tout semble dit en son nom, tout semble être vrai comme l'Évangile. »]. Voir A. J. Minnis, « Theorizing the Rose : Commentary Tradition in the Querelle de la Rose ", Poetics. Theory and Practices in Medieval English Literature, dir. Piero Boitani et Anna Torti, Cambridge, D. S. Brewer, 1991, p. 13-35; R. Brown-Grant, "A New Context for reading the Querelle de la Rose: Christine de Pizan and Medieval Literary Theory ", Au champ des escriptures, III colloque international sur Christine de Pizan, dir. Éric Hicks, Paris, Honoré Champion, 2000, p. 581-595.

39. J. de Montreuil, épître Ut sunt mores, dans Débat, éd. Hicks, p. 42 : « Nec quod demum satirici is instructor fungitur officio, quo respectu plura licent que aliis actoribus prohibentur.» ["Ils méconnaissent la tâche de satiriste de cet auteur, grâce à laquelle des choses interdites à d'autres lui ont été permises. »] Dante, auteur dont discutent les débatteurs de la Rose, avait déclaré que la personnification était une «figure ou ornement rhétorique accordée aux poètes » (Vita nova, éd. et trad. M. Marietti, Cr. Tullio Altan et J.-Ch. Vegliante, Paris, Classiques Garnier, 2011, p. 97).

40. Dans son excuse aux lectrices, Jean de Meun rejette la responsabilité des propos misogynes sur les textes qu'il cite (Le Roman de la Rose, op. cit., p. 802, v. 15222-24) : « Mais as aucteurs vous en prenez/ qui en leur livres ont escrites/ les paroles que j'en ai dites. » [« Mais prenez-vous-en aux auteurs qui ont écrit dans leurs livres les paroles que j'ai dites. »]

41. P. Col à Chr. de Pizan, dans Livre des epistres, éd. Valentini, p. 339 [ « Il faut se souvenir de quel pied on boite pour marcher plus droit »]. Sur la fortune de cette image, voir L. J. Walters, «The foot on which he limps : Jean Gerson and the rehabilitation of Jean de Meun in Arsenal 3339 ", Digital Philology $\mathrm{n}^{\circ}$ 1/1, 2012, p. 110-138.

42. J. Gerson, sermon contre la luxure (décembre 1402), Euvres, t. VII*, op. cit., nº 370, p. 824.

43. Le Roman de la rose, op. cit., p. 564, v. 10569. 
44. Chr. de Pizan, épître à J. de Montreuil, dans Livre des epistres, éd. Valentini, p. 166 [« Mieux lui convient de disparaître dans le feu qu'être couronné de laurier »].

45. Chr. de Pizan à P. Col, dans ibid., p. 185 [« Sais-tu comment il en va de ce genre de lecture? Comme avec les livres des alchimistes : les uns les lisent et les comprennent d'une manière, les autres comprennent tout le contraire, et chacun croit avoir trouvé la bonne interprétation. Et les voilà qui allument leurs fourneaux, leurs alambics et leurs creusets, qui mélangent des métaux et matières diverses, et qui soufflent de toutes leurs forces. Et s'ils trouvent un brin de sublimation ou de précipitation, ils croient avoir découvert quelque chose d'extraordinaire. [...] Il en va de même pour toi, moi et quelques autres. Tu lis et tu comprends d'une manière et moi de la manière inverse ; tu affirmes et je réplique. Et une fois que nous avons fait ceci et cela, tout cela ne vaut rien parce que le sujet de notre discussion est inconvenant et que nous sommes comme les alchimistes qui croient faire de l'or avec du fumier. »].

46. Le débat a joué un rôle majeur dans la carrière publique de Christine; voir Chr. de Pizan, Le Livre de l'advision Cristine, éd. Chr. Reno et L. Dulac, Paris, Honoré Champion, 2001, p. 165 : «Et comme je tiens pour la chose non usagee que femme escripse, [...] ainsy furent en peu de heure ventilez et portez mes livres en pluseurs pars et pays divers. » [ Comme je suppose qu'il n'est pas courant qu'une femme écrive, en peu de temps mes livres furent diffusés dans de nombreuses régions et pays divers. »]

47. G. Col à Chr. de Pizan, dans ibid., p. 170 ; P. Col à Chr. de Pizan, dans ibid., p. 327.

48. Elle apparait sous les traits de « dame Raison la sage » parmi les juges du procès de la Rose mis en scène dans le Traité (dans Livre des epistres, éd. Valentini, p. 299). Elle s'oppose à la Bouche, écolière dissipée et lectrice de Jean de Meun, dans l'un des sermons prêchés à Paris pendant l'hiver 1402 (Euvres..., t. VII*, op. cit., p. 838).

49. J. de Montreuil, épître Ut sunt mores, dans Débat, éd. Hicks, p. 44 ; P. Col, dans Livre des epistres, éd. Valentini, p. 329.

50. Ibid., p. 347.

51. Jean de Montreuil, épître Quo magis, dans Débat, éd. Hicks, p. 30 : «Non adeo feroces sumus ut que sit in disputando libertas ignoremus. " ["Nous ne sommes pas si intransigeants que nous ignorions dans quel esprit de liberté il convient de discuter. »]

52. Gerson, Talia de me, dans Débat, éd. Hicks, p. 162 : «Professio mea debens erroribus et viciis quantum valet obniti » [ "Ma profession m'oblige à lutter constamment contre les erreurs et les vices »]. Sa vision de la rhétorique chrétienne, incarnée par Éloquence théologienne, reprend explicitement les préceptes du De Doctrina Christiana de saint Augustin.

53. Gerson, Traité, dans Livre des epistres, éd. Valentini, p. 323 : «Rien je ne conclus contre la personne de l'aucteur [...] ; mais du deffault, qui est trop grant, je parle. »

54. Ibid., p. 310 [ «Si quelqu'un se déclarait ennemi du roi de France et sous ce nom et cette identité lui faisait la guerre, est-ce que ce nom l'empêcherait d'être considéré comme un traitre et mis à mort? Si prenant le rôle d'un hérétique ou d'un sarrasin, voire du diable, quelqu'un écrit et diffuse des erreurs contre la foi chrétienne, en sera-t-il excusé ?»].

55. Ibid., p. 321.

56. P. Col à Chr. de Pizan, dans ibid., p. 342 : «Je cognois home fol amoureux, le quel, pour soy oster de fole amor, a emprunté de moy le Ronmant de la rose, et luy ay oy jurer de sa foy que c'est la chose qui plus li a aidié a s'en oster. » [ "Je connais un homme obsédé par le mauvais amour qui, pour s'en guérir, m'a emprunté le Roman de la rose ; il m'a juré sur sa foi que c'est ce qui l'a le plus aider à s'en sortir. »]

57. Chr. de Pizan à P. Col, dans ibid., p. 198 [ «Un homme marié qui croit au Roman de la rose comme à l'Évangile. [...] Et à chaque parole qu'il trouve à son goût, il la frappe d'un ou deux coups de pied ou de poing. Il me semble que, si certains s'en louent, cette pauvre femme le paie bien cher. »]. 
58. Gerson, sermon sur la luxure, Euvres.., t. VII*, op. cit., nº 371, p. 839. Au début du débat, Christine de Pizan a abordé la question de la réception sous un angle social et genré (épître à J. de Montreuil, dans Livre des epistres, éd. Valentini, p. 164) : les «deshonnestes ficcions " de la Rose provoqueraient la gêne des lectrices de cour, « roynes, princeces et vaillans preudefemmes ».

59. Gerson, Traité, dans ibid., p. 313 [«Au feu, bonnes gens, au feu! [...] Celui qui ne fuit pas le danger trébuchera et sera pris comme le rat au morceau de lard et le loup dans le piège, le papillon à la flamme de la chandelle, les fous ou les enfants attirés par la beauté des épées tirées ou des charbons ardents. »].

60. Cicéron, De l'orateur, III, 53, 205, éd. Henri Bornecque et Edmond Courbaud, Paris, Les Belles Lettres, 1971, t. III, p. 85.

61. Chr. à J. de Montreuil, dans Livre des epistres, éd. Valentini, p. 166.

62. Gerson, Traité, dans ibid., p. 323.

63. Ibid., p. 315 ; «a re publica christiane religionis exulandas » (épître Talia de me à Pierre Col, dans Débat, éd. Hicks, p. 162). Sur la relation de la Rose à l'hérésie, voir A. Leupin, « L'hérésie littéraire. Paradigmes textuels dans le Roman de la rose ", De la Rose, Textes, Image, Fortune, dir. C. Bel et H. Braet, Louvain, Peeters, 2006, p. 59-80.

64. Gerson, sermon sur la luxure (décembre 1402), Eeuvres, t. VII*, op. cit., n 371, p. 839 ["Si j'avais un unique exemplaire du Roman de la rose et qu'il coûtait mille livres, je le brûlerais plutôt que de le vendre; si j'apprenais que Jean de Meun ne s'est pas repenti de sa rédaction, je ne prierais pas plus pour lui que pour Judas ; si j'entendais en confession quelqu'un qui serait incité par le roman à mal se comporter, je lui ordonnerais d'en censurer plusieurs épisodes ou de jeter le tout »].

65. Voir K. Sullivan, «The Inquisitorial Origins of Literary Debate ", Romanic review $\mathrm{n}^{\circ} 88,1997$, p. 27-51; la dimension inquisitoriale du débat a été nuancée par H. Solterer, «Fiction versus defamation: the quarrel over the Romance of the Rose », Medieval History Journal n ${ }^{\circ} 2 / 1,1999$, p. 111-141.

66. L'âge des orateurs en français (1390-1560) est étudié dans deux projets sous ma direction, L'Âge des orateurs sur la période 1390-1460 (IUF, 2015-2018) et Médialittératures sur 1460-1560 (FNS, 2020-2024).

67. La dernière lettre de P. Col à Chr. de Pizan, fin 1402, est restée inachevée, probablement sous la pression de Gerson qui a invité Col à ne plus donner " occasion de scandale à des innocents » (dans Débat, éd. Hicks, p. 175).

68. La première vague de publications américaines sur le Débat est contemporaine des lois McKinnon sur la pornographie (1993-2000) ; voir entre autres D. Hult, «Jean de Meun's Romance of the Rose and the Hermeneutics of Censorship ", New Literary History n ${ }^{\circ} 28 / 2,1997$, p. 345-366.

69. Gerson, Traité, dans Livre des epistres, éd. Valentini, p. 304-305; réponse à Pierre Col, épître Talia de me, dans Débat, éd. Hicks, p. 175.

70. Gerson, Traité, dans Livre des epistres, éd. Valentini, p. 304 : «Eloquence, comme saige et bien apris [...] en guise d'un homme aucunement pensif» [ Éloquence, en homme sage et bien éduqué, sous l'apparence d'un homme quelque peu pensif »]; Chr. de Pizan à P. Col, dans ibid., p. 191 « Tu respons a dame Eloquence et moy que maistre Jehan de Meun, en son livre, introduisi personnages....».

71. E. Doudet, «Christine de Pizan et l'orateur au féminin au $\mathrm{XV}^{\mathrm{e}}$ siècle », L'Auctorialité au féminin dans les fictions courtoises, des trobairitz à Christine de Pizan, dir. N. Koble, A. Arato et R. Décloître, Fabula Colloques en ligne, 2018 ; URL : https://www.fabula.org/colloques/document6265.php. 


\section{AUTEUR}

\section{ESTELLE DOUDET}

Université de Lausanne - Université Grenoble Alpes

Institut universitaire de France 